\title{
Human brain shaped by duplicate genes
}

\section{Multiple copies of a gene may have boosted the computational power of our ancestors' brains.}

\section{Ewen Callaway}

03 May 2012

Humans walk on two feet and (mostly) lack hair-covered bodies, but the feature that sets us furthest apart from other apes is a brain capable of language, art, science, and other trappings of civilisation.

Now, two studies published online today in Cell ${ }^{1,2}$ suggest that DNA duplication errors that happened millions of years ago might have had a pivotal role in the evolution of the complexity of the human brain. The duplications - which created new versions of a gene active in the brains of other mammals - may have endowed humans with brains that could create more neuronal connections, perhaps leading to greater computational power.

The enzymes that copy DNA sometimes slip extra copies of a gene into a chromosome, and scientists estimate that such genetic replicas make up about $5 \%$ of the human genome. However, gene duplications are notoriously difficult to study because the new genes differ little from their forebears, and tend to be overlooked.

Evan Eichler, a geneticist at the University of Washington in Seattle, and lead author of one of the Cell papers, previously found that humans have four copies of a gene called SRGAP2, and he and his colleagues decided to investigate.

In their new paper, they report that the three duplicated versions of SRGAP2 sit on chromosome 1, along with the original ancestral gene, but they are not exact copies. All of the duplications are missing a small part of the ancestral form of the gene, and

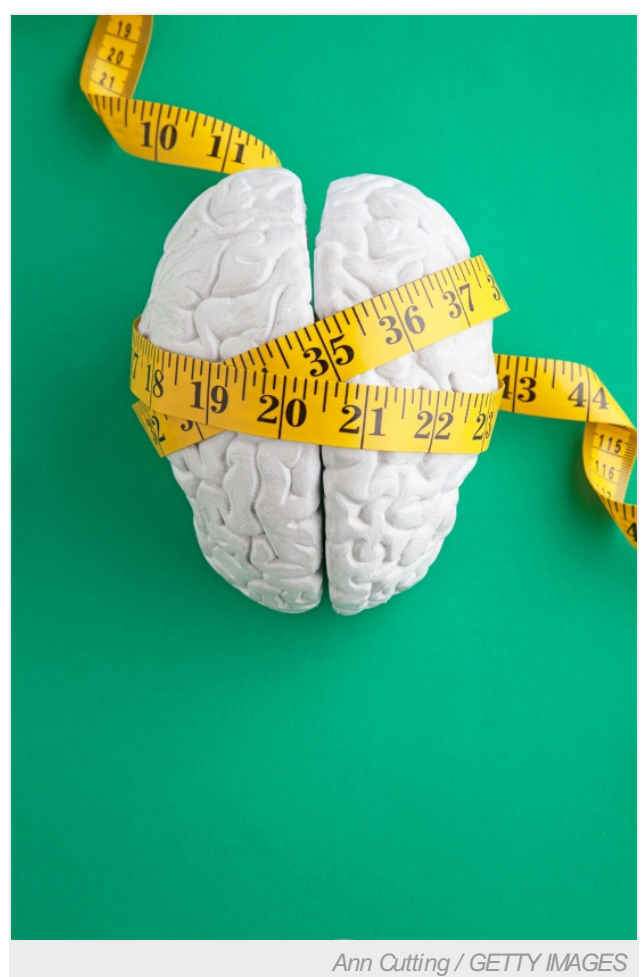

Could duplicated genes explain why you can read this? at least one duplicate, SRGAP2C, seems to make a working protein. Eichler's team has also found SRGAP2C in every individual human genome his team has examined - more than 2,000 so far - underscoring its significance.

"Ten years after the human genome was sequenced and declared done, we're still finding new genes in new places that are really important to human brain function and evolution," says Eichler.

Eichler's team calculates that SRGAP2C appeared roughly 2.4 million years ago, around the time that big-brained species of Homo evolved in Africa from smaller-skulled Australopithecines, and around the time that stone tools appeared in the fossil record. These ancient hominins eventually gave rise to Homo erectus, which were the first human ancestors to wander beyond Africa, roughly 1.8 million years ago.

\section{Boosting brains}

According to a second study, also published in Cell ${ }^{2}$, the emergence of SRGAP2C could have helped our ancestors to boost the power of their bigger brains, which may have been created by other, unknown changes in the genome.

"It's got to play some important function," says Franck Polleux, a neurobiologist at the Scripps Research Institute in La Jolla, California, and senior author of the second paper in Cell.

Surprisingly, the SRGAP2C protein blocks the action of the ancestral protein, Polleux's team discovered, effectively rendering humans as 'knockouts' for the ancestral SRGAP2 gene. The team then expressed the human form of SRGAP2C in the neurons of developing mice. The change didn't cause the mice brains to enlarge, but their neurons produced denser arrays of brain cell structures, called dendritic spines, that forge connections with neighbouring neurons. 
"If you're increasing the total number of connections, you're probably increasing the ability of this network to handle information," Polleux says. "It's like increasing the number of processors in a computer."

In mice, the gene also increased the migration speed of neurons across the developing brain. Polleux's team speculates that this trait could also have helped neurons to travel long distances in the enlarged brains of human ancestors.

"One has to be cautious about putting too much emphasis on the role of one gene in brain evolution," says Genevieve Konopka, a neuroscientist at the University of Texas Southwestern Medical Center in Dallas. But she thinks that the two papers make a good case that duplication of SRGAP2 influenced human cognition.

James Sikela, an evolutionary geneticist at the University of Colorado, Denver, adds that the SRGAP2 duplications are likely to be one of a multitude of genetic changes that moulded the human brain. His team has identified dozens of duplicated genes unique to humans ${ }^{3}$, many of them expressed in the brain. "Finding the genes that make us human may be challenging," he says, "but the resources we now have to ask such questions are unprecedented."

Nature | doi:10.1038/nature.2012.10584

\section{References}

1. Dennis, M. Y. et al. Cell http://dx.doi.org/10.1016/j.cell.2012.03.033 (2012).

2. Charrier, C. et al. Cell http://dx.doi.org/10.1016/j.cell.2012.03.034 (2012).

3. Fortna, A. et al. PLoS Biology 2, e207 (2004). 